\begin{tabular}{|c|l|}
\hline Title & Linguistische Ästhetik : zum mimetischen und logischen Gebrauch der Schrift \\
\hline Sub Title & \\
\hline Author & Stetter, Christian \\
\hline Publisher & 慶應義塾大学日吉紀要刊行委員会 \\
\hline Publication year & 2001 \\
\hline Jtitle & 慶應義塾大学日吉紀要. ドイツ語学・文学 No.32 (2001. 3),p.72- 95 \\
\hline JaLC DOI & \\
\hline Abstract & \\
\hline Notes & \\
\hline Genre & Departmental Bulletin Paper \\
\hline URL & https://koara.lib.keio.ac.jp/xoonips/modules/xoonips/detail.php?koara_id=AN100 \\
32372-20010331-0072
\end{tabular}

慶應義塾大学学術情報リポジトリ(KOARA)に掲載されているコンテンツの著作権は、それぞれの著作者、学会または出版社/発行者に帰属し、その 権利は著作権法によって保護されています。引用にあたっては、著作権法を遵守してご利用ください。

The copyrights of content available on the KeiO Associated Repository of Academic resources (KOARA) belong to the respective authors, academic societies, or publishers/issuers, and these rights are protected by the Japanese Copyright Act. When quoting the content, please follow the Japanese copyright act. 


\section{Linguistische Ästhetik: Zum mimetischen und logischen Gebrauch der Schrift}

\section{Christian Stetter}

Die Praxis der Sprachwissenschaft ist seit jeher eine literale gewesen. Man beschrieb und erklärte die Sprachen oder die Sprache, indem man sich des Alphabets oder ähnlicher Schrifttypen bediente, um die zu beschreibenden Sprachformen zu vergegenständlichen.') Die Frage, was der Sprachwissenschaftler tut, wenn er im Rahmen seiner Arbeit Schrift benutzt, ist daher lange Zeit im Hintergrund der erkenntnistheoretischen und methodologischen Debatten geblieben, die die Entwicklung der Linguistik begleitet haben. ${ }^{2}$ Erst im Kontext der Entdeckung der Schrift als genuinen sprachwissenschaftlichen Themas gewinnt sie allmählich Kontur. Gestellt wurde sie zunächst als technische und methodische Frage, dann als methodologische, z. B. im Zusammenhang mit dem Transkriptionsproblem. Ihre Brisanz entfaltet sie aber erst, wenn man sie grundsätzlich nimmt, also fragt, was die literale Praxis der Sprachwissenschaft für das ,Sein` ihrer Gegenstände bedeutet. Dies will ich im folgenden tun, um daraus in einem zweiten Schritt einige konzeptionelle Konsequenzen für die Linguistik zu entwickeln.

1) Man braucht nicht unbedingt bis zu Panini zurückzugehen, um dies zu konstatieren. Wenn ich von Sprachwissenschaft spreche, so beschränke ich mich auf die moderne Sprachwissenschaft, als deren Gründervater man sicher W. von Humboldt ansehen kann, auch wenn die sprachphilosophische Essenz seiner Konzeption vom linguistischen Mainstream des 19. Jahrhunderts nicht wahrgenommen wird. Vgl. hierzu Stetter 1997, Kap. 10.

2) Noch in dem 1979 von Jäger herausgegebenen Band Erkenntnistheoretische Grundfragen der Linguistik wird die Frage nach der literalen Praxis der Linguistik überhaupt nicht berührt. Vgl. Jäger 1979. 
Wenn der Linguist im Rahmen seiner Arbeit Schriftsysteme benutzt, so tut er dies dem traditionellen Verständnis linguistischer Praxis zufolge erstens qua Wissenschaftler. Erst die schriftliche Fixierung seiner Beschreibungen, Rekonstruktionen usw. - wie immer man die Resultate seiner Arbeit bezeichnen will - sichert deren empirische Nachprüfbarkeit und die logische Kontrolle seiner Behauptungen. Wissenschaftliche Theorien sind nur im Medium der Schrift möglich. Zweitens tut er dies qua deskriptiver Wissenschaftler: Er verwendet Wörter als Begriffe, d. h. in logischem Sinn. Man kann von ihm verlangen, daß er die Intension seiner Begriffe hinreichend genau definiert und daß er keine leeren Begriffe verwendet. ${ }^{3)}$ Die von ihm verwendeten geschriebenen Wörter haben also eine mehr oder weniger explizit definierte Semantik, die sich hierin von der fluktuierenden Gebrauchssemantik der oralen Sprache kategorial unterscheidet. Als Linguist schließlich ,rekonstruiert' er ein sprachliches System oder Teile eines solchen Systems, indem er begrenzte Auswahlen von Performanz-Daten beschreibt, miteinander vergleicht, ,typische' von ,untypischen' (kontingenten, nicht kategorisierbaren, ...) Zügen unterscheidet, Fragmente des ,Typischen“ zusammenfügt usw. ${ }^{4)}$

Die Schrift erscheint in dieser Konzeption als ,neutrales' Medium in folgendem Sinn:5) Der (linguistische) Text wird verstanden als Folge von Namen, Prädikatoren und synkategorematischen Zeichen, und wenn die Arbeit des Linguisten auch schriftlich vor sich gehen muB, so scheint doch der Kern des logischen Geschäfts, der Modus des Behauptens nicht von der Frage tangiert, ob er nun mündlich, schriftlich oder auf sonstige symbolische Weise vollzogen wird. Und zweitens scheint die Wahl des betreffenden Schrifttyps oder der jeweiligen Sprache irrelevant. Daß die lingua franca der Linguistik im 20. Jahr-

3) Letzteres scheint selbstverständlich, doch lebt die ganze linguistische Universalienforschung davon, daß diese Frage ausgeblendet bleibt. Referentialisierbar sind Aussagen über vermeintliche Universalien immer nur auf dem „Umweg“ über die Ersatzreferentialisierung mittels Ausdrücken, die einer bestimmten "Einzelsprache“ angehören. Vgl. hierzu Stetter 1999a.

4) Vgl. Goodman 1984, S. 124 ff.

5) Vgl. zum Problem der Medienneutralität Krämer 1998. 
hundert das Englische geworden ist, wird ersichtlich als pragmatische Folge der Globalisierung der Wissenschaftskommunikation gedeutet. Physiker und Mediziner kommunizieren international längst schon auf Englisch. Allein bei den Philosophen scheint sich noch die Position zu halten, daß man Platon „eigentlich“ nur vom griechischen, Wittgenstein nur vom deutschen Originaltext her verstehen könne. ${ }^{6)}$

$\mathrm{Da}$ sich für die Linguistik hier ein besonderes Problem ergeben könnte, zeigt sich jedoch, sobald man deren empirischer Kernfrage etwas genauer nachgeht: Wie legt man fest, was eine sprachliche Einheit ,ist'? Z. B. ein Subjekt-von ..., ein Verb, eine Silbe, ein Phonem, ... Der Linguist befindet sich hier in einer grundsätzlich anderen Situation als der Philologe. Für diesen stellt sich die Frage nicht. Er hat seinen Text je vor sich, und er muß, wenn schon, dann das Subjekt oder das Verb dieses Satzes, die Silben dieses bestimmten Wortes hier bestimmen, und mit Phonemen hat er es per se nicht zu tun. Der Linguist dagegen ist nicht am Text interessiert, nicht an dem, was diesen Text - nehmen wir Platons 7. Brief - zu einem Individuum unter allen Texten macht, was z. B. gestattet, ihn als „echt“ anzuerkennen. Vielmehr an dem - um beim Beispiel zu bleiben -, was sich ihm an allgemeinen Erkenntnissen über das attische Griechisch des 4. vorchristlichen Jahrhunderts ablesen läßt, seinen lexikalischen Bestand, seine Morphologie, seine Syntax. Er handelt also über ein Objekt, das phänomenal nie in Erscheinung tritt, das weder räumlich noch zeitlich $\mathrm{zu}$ verorten ist - weder hat Platons Griechisch nur in Athen oder Attika existiert, sondern war präsent, wo immer jemand Platons Griechisch sprach, noch hat es zu einer bestimmten Zeit begonnen oder aufgehört zu existieren. Und doch soll es sich dabei um ein empirisches Objekt handeln. Wir unterstellen wie selbstverständlich, daß der Begriff „das Griechisch Platons" nicht leer ist und daß wir mit dem zitierten Ausdruck auf etwas Bestimmtes Bezug nehmen, das der Altphilologe von anderen koexistierenden Sprachen oder vom Griechisch Homers oder dem des Neuen Testaments anhand ausweisbarer Kriterien zu unterscheiden weiß. Der Linguist nimmt also sogenannte Performanzereig-

6) Mir ist jedenfalls keine intralinguistische Debatte über dieses Problem bekannt. Die Praxis ist zu selbstverständlich. 
nisse als Beispiele für ,typische' Gebräuche, rekonstruiert, um bei der saussureschen Terminologie zu bleiben, aus Phänomenen der parole das hinter diesen „liegende“ System, die langue, die - so die metaphysische Grundannahme, die die Linguistik als Disziplin konstituiert ${ }^{7)}-$ die parole erst möglich macht.

An der sogenannten Lexikonregel einer Phrasenstrukturgrammatik läßt sich das Problem, das wir hier verfolgen, exemplarisch studieren: Bekanntlich hat sie die folgende-oder eine äquivalente-Form: $\mathrm{X}\{a b c d, e f g h, i j k l, \ldots\}$. Einer syntaktischen ,Endkategorie wird als seine Extension eine Menge von Ausdrücken zugeordnet. Die vermeintliche Regel ist also keine Regel. ${ }^{8)}$ Die logisch korrekte Lesart der Lexikonregel lautet vielmehr: abcd ist ein $\mathrm{X}, e f g h$ ist ein $\mathrm{X}, i j k l$ ist ein $\mathrm{X}$, ...9) Die drei Pünktchen, die sich am Ende jeder Lexikonregel finden sofern es sich um eine Grammatik handelt, die eine natürliche Sprache beschreibt-bedeuten weit mehr, als daß sich der Schreiber dieser Grammatik das ihn und den Leser derselben langweilende Geschäft erspart hätte, die angefangene Reihe zu vervollständigen. Tatsächlich sind sie das philosophisch Interessanteste an dem ganzen Ausdruck, Symbol bzw. Kaschierung des Kunststücks, mit dem Saussure die Linguistik als systematische Disziplin etablieren konnte: der Unterscheidung von Synchronie und Diachronie.

So notwendig diese Unterscheidung ist, sie ist doch je nur eine vorläufige. Sie gibt dem Sprachwissenschaftler die Zeit, die er braucht, um von dem langsam, aber kontinuierlich und unaufhaltsam sich wandelnden Objekt, das er beschreiben will, eine Momentaufnahme zu fertigen: So sieht die Syntax der deutschen Gegenwartssprache am Ende des 2. Jahrtausends aus, könnte sich der Autor des Grundrisses der deutschen Grammatik gesagt haben, als er nach mühevollen Jahren die Arbeit an diesem Werk abschloß. Doch er wußte, daß dies eine Illusion war, und hat dies auch in seinem Vorwort zum Ausdruck gebracht: Die Arbeit des Linguisten, ein Sprachsystem zu beschreiben,

7) Vgl. hierzu Stetter 1997, Kap. 3.

8) Denn die logische Form einer Regel ist ein generalisiertes Konditional. Dieses liegt hier aber nicht vor.

9) Das Komma hat die logische Bedeutung des nicht ausschließenden "oder". 
gleicht der Arbeit des Malers, der beauftragt wurde, die Golden Gate Bridge neu zu streichen, weil man an deren südlichem Ende Rostspuren entdeckt hatte. Als er nach etlichen Jahren am anderen Ende angelangt war, zeigten sich am südlichen Rostspuren. ${ }^{10)}$ Der Autor wußte in philosophischem Sinn - wovon er schrieb. Die drei Pünktchen sind Ausdruck der Tugend, die der Linguist aus seiner Not macht: Anstatt sich in dem vergeblichen Versuch zu erschöpfen, hic et nunc eine möglichst vollständige Liste auch nur der Präpositionen oder Konjunktionen, gar der Verben der deutschen Gegenwartssprache zu erstellen, gibt er Beispiele, die dem sprachkundigen und verständigen Leser ${ }^{11}$ der Grammatik Hinweise darauf geben, was für den Schreiber der Grammatik ein $\mathrm{X}$, ein $\mathrm{V}[\mathrm{erb}$, eine $\mathrm{K}$ [onjunktion], eine $\mathrm{P}[$ räposition] usw. ,ist'. Verstehen wird der Leser diese Hinweise aber nur, wenn er imstande ist, die Buchstabenfolgen, die in dem dem jeweiligen Kategorienausdruck zugeordneten Mengenausdruck aufgeführt sind, als Darstellungen von Wörtern der betreffenden Sprache zu lesen. ${ }^{12)}$ Dies aber heißt, daß hier die Linguistik von der Schrift einen zweifachen Gebrauch macht. Betrachten wir dazu die explizite Form einer Instantiierung einer Lexikonregel, das Konstativum

10) Vgl. Eisenberg 1989, S. 9.

11) Verständig muß der Leser einer Grammatik sein, damit er die abkürzenden, die Metaphorik - die ja ebenso zum „normalen“ Sprachgebrauch gehört wie das Regelmäßige - vernachlässigenden Darstellungen „seiner“ Sprache sozusagen nicht bierernst nimmt, für nicht mehr, als sie sein können: eine mühsame Annäherung an eine unbekannte Realität.

12) Dies ist keineswegs selbstverständlich, sondern Resultat einer speziellen Schulung im Umgang mit formalen Darstellungen von Sprachgebräuchen. Hier wird ein Registerwechsel erheblichen Ausmaßes verlangt: die Fähigkeit der Übertragung theoretischen Wissens auf ein seiner Natur nach weitgehend unreflektiertes Können.

Anmerkung zur Anmerkung: Wir stoßen bei diesen Erörterungen immer wieder auf das philosophische Problem des sogenannten "tacit knowledge“, des sprachlichen "Wissens", das der Doktrin der generativen Linguistik zufolge Charakteristikum der Sprachkompetenz sein soll. M. E. hat H. J. Schneider (1992, S. 64 ff.) die problematischen Punkte in dieser Konzeption so klar bezeichnet, daß ich im folgenden das Problem übergehe, auch dort, wo sachlich ein Kommentar angebracht wäre. 
"geben“ ist ein Verb.

Wir haben hier erstens den üblichen logischen Gebrauch, den wir von qua Buchstabenfolgen dargestellten Wörtern machen: Referenz und Prädikation. ${ }^{13)}$ Mit "geben" referieren wir auf das Objekt des Konstativums, mit ist ein Verb sprechen wir diesem ein Prädikat zu. Nun beachte man die Form des Namens, mittels dessen die Referenz vollzogen wurde bzw. wird. Sie lautet "geben“, nicht, wohlgemerkt, geben. Die Anführungszeichen - oder äquivalente typographische Mittel sind unverzichtbar, denn geben ist ein Verb wäre erstens eine ganz andere Behauptung als die hier betrachtete, und zweitens eine offenkundig absurde, ${ }^{14)}$ während "geben" ist ein Verb ebenso offenkundig eine nicht nur nicht absurde, sondern zudem allgemein anerkannt zutreffende Behauptung ist. Also müssen die Anführungszeichen eine hier relevante Bedeutung haben.

Der logisch grundlegende Sinn des Gebrauchs von Anführungszeichen besteht darin, klarzumachen, daß der zitierte Ausdruck an der betreffenden Stelle nicht im üblichen Sinn zu lesen, sondern daß der Ausdruck selbst Objekt des betreffenden Diskursausschnitts ist. ${ }^{15)}$ Hier heißt dies - das allgemeine logische Problem des Zitierens brauchen wir nicht zu betrachten -, daß die Buchstabenfolge "geben“ nicht in logischem Sinn verwendet wird, nicht als Begriff mit einer bestimmten Semantik, sondern als Darstellung, lateinisch exhibitio, des Wortes "geben“ qua Wort der deutschen Sprache.

Man macht hier also von den Anführungszeichen einen besonderen Gebrauch. Es handelt sich durchaus nicht um den üblichen Fall des Zitie-

13) Vgl. Austin 1975, S. 93 und $98 \mathrm{f}$.

14) Die Absurdität beruht auf einem Kategorienfehler.

15) Darum werden Anführungszeichen in diesem logischen Gebrauch immer nur in Satzkonstituenten verwendet, mittels derer auf etwas referiert wird. Dies im Unterschied zum rhetorischen Gebrauch von Anführungszeichen, durch den man den Leser gleichsam bittet, er möge den Gebrauch des in Anführungszeichen gesetzten Wortes hier hinnehmen, so wenig genau er auch sein möge, z. B.: Im "normalen" Sprachgebrauch wird dieses Wort so und so verwendet. Was ist denn schon, könnte man pingelig fragen, der "normale" Sprachgebrauch? 
rens. Dieses hat den Zweck, einen bestimmten Ausdruck, ein Wort oder eine Folge von Wörtern, in den aktuellen Diskurs einzuführen, um darüber etwas aussagen zu können:

Das Wort "geschichtlich“ bedeutet in der Sprache Wilhelm von Humboldts ...

Das Wort "geschichtlich“ in der Sprache Wilhelm von Humboldts ist hier das Objekt des Konstatierens, es geht genau um dieses und um sonst nichts. Dieses Wort, nicht seine Gestalt etwa, ist der Gegenstand, über den metasprachlich etwas behauptet, d. h., der unter einen Begriff subsumiert wird. Bei „geben“ ist ein Verb oder - um das Problem noch klarer zu machen - bei „abcd“ ist ein $X$ liegt der Fall aber anders: Die Ausdrücke „geben“ bzw. „abcd“ haben hier ja den Sinn, anhand dieses Beispiels klarzumachen, was der Ausdruck „Verb“ bzw. „X“ im fregeschen Sinn ,bedeuten“ soll, d. h. die Extension von „Verb“ bzw. „X“ andeutungsweise zu erhellen. Ohne diese und andere Beispiele könnte man sich überhaupt keinen Begriff davon machen, welchen (fregeschen) Sinn, d. h. welche Intension der Begriff „Verb“ bzw. „X“ hier hat. Also hat hier die Anführung des Ausdrucks "geben“ - um nun wieder bei diesem zu bleiben - den Zweck, dem Leser eine Zeichengestalt vor Augen zu führen, die er, so er der deutschen Sprache mächtig ist, als ein Exemplar des ihm bekannten Wortes "geben“ zu identifizieren vermag, um so zu dem Schluß zu kommen, daß der linguistische Begriff des Verbs offenbar nicht leer ist. ${ }^{16)}$

Der in der Lexikonregel zitierte Ausdruck ist ein Muster dessen, was wir das deutsche Wort „geben“ nennen. Und er exemplifiziert dieses Wort auch nur in bestimmten Hinsichten. Es ist so wenig dieses Wort wie das Teppichmuster, das uns der Händler bei der Wahl unseres Teppichbodens vorlegt, der Teppichboden wäre, den wir schließlich haben werden. Exemplifikation ist nach Goodman die (exhibitive) Darstellung einer Eigenschaft durch Bezugnahme auf diese. ${ }^{17)}$ Ein Muster

16) Die Intension linguistischer Kategorien wird dagegen in Konstitutionsund Dependenzsystemen beschrieben.

17) Vgl. Goodman 1997, S. 59 ff. 
eines Stück Stoffs kann diesen als fest gewebten oder roten nur exemplifizieren, sofern in der Handlung des Exemplifizierens darauf Bezug genommen wird: Fühlen Sie, wie fest dieser Stoff gewebt ist; diesen Rot-Ton hat der Stoff, vergleichen sie ihn mit anderen usw.

In welcher Hinsicht exemplifiziert also das Muster "geben" das Wort „geben“? Nur in Hinsicht auf die interne Artikulationsweise der Zeichengestalt, und zwar hier der schriftlichen. Dem Leser wird die Buchstabenfolge $g+e+b+e+n$ vor Augen geführt. So schreibt man eben das Wort "geben“, so und nicht anders sieht es aus, nota bene: geben, nicht $g+e+b+e+n .{ }^{18)}$ Hätte sich nun der Schreiber des Exempels vertan und statt $g+e+b+e+n$ die Buchstabenfolge $b+e+b+e+n$ geschrieben, so würde der Leser das Beispiel als Exempel des Wortes "beben“ nehmen, und bei „veben“ oder „feben“ - alles buchstäblich naheliegende Tippfehler - würde er als des Schriftdeutschen mächtiger Leser - gar nichts verstehen. Der Rekurs auf das Mündliche würde nicht helfen. Die Bedeutung des Zeichens "geben“ wird nur in dem Medium erzeugt, in dem das Zeichen artikuliert wird: hier eben in der Schrift. ${ }^{19)}$ Gerade deshalb würde das Entsprechende in gleicher Weise gelten, wenn jemand, z. B. im Grammatikunterricht, sagte, "geben“ ist ein Verb. Dann wäre das, was die Schüler vernehmen, ein Beispiel für einen Ausdruck, der hier hinsichtlich seiner Artikulationsweise exemplifiziert wird, die hier eben die mündliche wäre. Bei solcherart exemplifizierender Darstellung von Wörtern oder anderen sprachlichen Ausdrücken machen wir von den artikulatorischen Einheiten

18) $g+e+b+e+n$ ist die exhibitive Darstellung einer Buchstabenfolge, nicht eines Wortes.

19) Notwendige Bedingung für das Verstehen der Handlung des Exemplifizierens ist nur, daß der Leser die ihm gegebene Buchstabenfolge "geben" als ein Wort $z u$ identifizieren vermag, das er lesen kann. Ihm muß dabei nicht klar sein, daß diese Buchstabenfolge ein - zudem nur analoges Modell der Artikulationsweise des oralen Wortes ist. Die Rückkopplung von geschriebener und gesprochener Sprache gibt es in allen Schriftsystemen, sie funktioniert aber nur auf der Ebene des Wortes, nie auf der subsemantischen Ebene. Dies ist einer der klassischen Mythen, die die Alphabetschrift von Anfang an begleitet haben. Er beruht auf der Verwechslung von Funktions- und Konstitutionsprinzip dieses Schrifttypus. Vgl. hierzu Stetter 1997, S. 56 ff. 
des betreffenden Sprachmediums also einen besonderen Gebrauch, den ich den ,mimetischen' Gebrauch nennen möchte. ${ }^{20)}$

Hinweise auf den Gebrauch der Zeichengestalt "geben“ gibt die Exemplifikation nicht. Darum stellt sie nicht das Wort dar, sondern "nur“ den signifiant. Denn das, was wir im - legitimen - naiven Sinne die Bedeutung des Wortes nennen, ist linguistisch betrachtet das Knowinghow seiner Verwendungsmöglichkeiten in syntaktischer wie semantischer Hinsicht. ${ }^{21)}$ Hiervon ist im Verfahren der Exemplifikation, wie es gerade beschrieben worden ist, aber abgesehen. Insofern ist der mimetische Gebrauch des Wortes etwas kategorial anderes als der logische. Dieser ist zudem der pragmatisch wie logisch primäre. Die Fähigkeit zum mimetischen Gebrauch eines Wortes, eines Ausdrucks oder wessen auch immer in Schrift oder mündlicher Sprache setzt immer die des logischen Gebrauchs voraus. Man muß das Wort verstanden haben, sonst kann man seine Zeichengestalt nicht identifizieren. ${ }^{22)}$

Damit stehen wir vor einem kardinalen kategorialen Problem der Linguistik. Es wird ihr von ihrer unverzichtbaren „internen“ schriftlichen Praxis aufgebürdet. Vielleicht ist es deshalb - meines Wissens jedenfalls - als solches bislang nie zur Kenntnis genommen worden. Materialiter hatten wir es oben schon gestreift: Wie stellt man schriftlich dar, wie man oraliter eine Wortform exemplifiziert, wie ist dies, ja ist dies überhaupt möglich? Betrachten wir dazu noch einmal das oben schon erörterte Beispiel:

20) Entsprechend der Bedeutung der Wörter $\mu i \mu \eta \sigma \iota s$ (mimesis) und $\mu i \mu \eta \mu \alpha$ (mimema) in Platons Kratylos (vgl. $430 \mathrm{~d}$ ff.).

21) Die regelmäßigen, nicht allerdings die metaphorischen Verwendungsmöglichkeiten eines Wortes sind in einer syntaktischen Beschreibung der betreffenden Sprache mehr oder weniger zutreffend und vollständig beschrieben. Insofern ist eine derartige Grammatik ein fragmentarisches Bild des betreffenden syntaktischen Knowing-how - eines Könnens, nicht dessen, was man in der generativen Linguistik sprachliches Wissen genannt hat. Vgl. z. B. Grewendorf, Hamm, Sternefeld 1987, kritisch zu diesem Sprachgebrauch Schneider 1992, S. 74 ff.

22) Beleg dafür ist die Funktion von Redundanzen in der Ausdifferenzierung der Gestalt sprachlicher Zeichen, die in geläufigem Gebrauch, schriftlich im Kursiven, mündlich in informeller Kommunikation, bis auf ein unverzichtbares Minimum abgebaut werden. 
... wenn jemand, z. B. im Grammatikunterricht, sagte, "geben“ ist ein Verb...

Diese Ausdrucksform ist literat Gebildeten so geläufig, daß man kaum auf den Gedanken kommt, hier könnte ein Problem liegen. Was den logischen Gebrauch der Schrift angeht, so gibt es hier auch keines. Denn berichtet wird hier lediglich, daß jemand sagte: „,geben“ ist ein Verb“. Jeder halbwegs geübte Leser wird das verstehen. Aber was besagt - betrachtet man die Sache aus der Perspektive des Exemplifizierens - der Ausdruck „... sagte, "geben“ ist ein Verb ..."? Hier ist nun das Zitat "geben" ist ein Verb ... nicht mehr Protokoll einer schriftlichen, sondern einer mündlichen Handlung des Exemplifizierens. Die Zeichengestalt "geben“ kann aber keine zutreffende Wiedergabe des exemplifizierten gesagten Ausdrucks sein, denn sie ist im Medium der Schrift gegeben, nicht im Medium der mündlichen Sprache - wie auch anders in einem schriftlichen Text. Zur Erinnerung: die relevante Hinsicht des Exemplifizierens war in diesem Fall die Artikulationsweise.

Den phänomenalen Unterschied von Schrift und oraler Sprache hat man stets mit der Opposition von flüchtig und dauernd beschrieben. Das ist und bleibt zweifellos zutreffend. Viele Unterschiede im Gebrauch und in der Funktionsweise beider Medien lassen sich auf diese Unterscheidung zurückführen. Doch muß man in der phänomenologischen Beschreibung der Differenz noch weiter gehen: Das schriftliche Zeichen existiert erst, wenn es geschrieben ist. Der Prozeß des Schreibens selbst - wie es geschrieben wird, nicht daß es geschrieben wird - ist für seine Geltung als Zeichen gleichgültig, solange die produzierte Gestalt nur im Rahmen der zulässigen Schemagrenzen bleibt. ${ }^{23)}$ Zum Zeichen wird diese erst aufgrund ihrer identifizierbaren Differenzen zu den Gestalten anderer Zeichen, seien diese nun Buchstaben oder Wörter. Die Identifizierung geschieht stets und nur in den Sprachspielen des Schreibens und Lesens in einem je gedachten formatierten Raum. ${ }^{24)}$ Viel deutlicher als in der Buchstabenschrift mit ihrem hohen

23) Deshalb kann man den Schreibprozeß auf technisch unterschiedlichste Weisen ins Werk setzen. Es kommt nur auf das Resultat an. In der mündlichen Kommunikation ist dies völlig anders.

24) Im Druck oder beim Schreiben auf dem PC ist der formatierte Raum 
Abstraktionsgrad wird dies in der Kalligraphie des Chinesischen oder Japanischen, etwa der Hiragana. Die ausbalancierte Eleganz dieser Zeichen, etwa des "o“ oder "mi“, wird man nur realisieren können, wenn man die Koordinaten des Raumes in seiner Vorstellung gegenwärtig hat, in den das Zeichen inskribiert werden soll. ${ }^{25)}$

Das gesprochene Wort dagegen fängt nicht an zu existieren, nachdem es artikuliert wurde, da existiert es schon nicht mehr. Es existiert genau dann und nur dann, wenn es artikuliert wird, genauer: indem es artikuliert wird. Die Essenz seiner Gestalt ist Bewegung, wie die des musikalischen Themas. Und diese Bewegung ist eine Einheit, deren formaler Ausdruck der Wortakzent ist. Der Instrumentalist lernt das Problem, von dem hier die Rede ist, technisch zu meistern: Selbst wenn im Presto von Beethovens op. 131 die erste Violine die gebundenen Halben des zweiten Takts aus technischen Gründen im Ab- und Aufstrich spielen muß, muß es doch wie gebunden klingen. Für die Beschreibung des analogen ästhetischen Problems der Artikulation der beweglichen, d. h. temporären Einheit, die das gesprochene Wort ist, fehlen der Linguistik bis dato jegliche Begriffe. ${ }^{26)}$ Es bleibt nur der Weg, sich dem Problem via negationis zu nähern: Das gesprochene Wort wird nicht durch ein Verfahren erzeugt, das der Aneinanderreihung von Buchstaben analog wäre, sondern durch Modulation des

technisch bereitgestellt, sodaß er dem Schreiber oder Leser bestenfalls bei außergewöhnlichen Layouts noch als notwendige Bedingung bewußt wird. Man erinnere sich aber daran, wie schwer es Schreibanfangern fällt, auf einem nicht linierten Papier leserlich zu schreiben.

25) Lehrbücher der Kalligraphie verdeutlichen daher diese gedachten Koordinaten durch punktierte Linien, die in etwa die Funktion haben der Linien in den Schreibheften der Erstklässler.

26) Dies ist natürlich auch ein - trauriges - Resultat des Faktums, daß die generative Linguistik mit ihrer Fixierung auf das Studium von Tiefenstrukturen das Studium der sogenannten "Oberfläche" sträflich vernachlässigt hat. Man hat Oberflächenelemente einfach als gegeben hingenommen, ohne weiter zu fragen, wie sie beschaffen sind. Man hatte sie ja schriftlich vorliegen. Der ganze Reichtum an Forschungsansätzen, den die strukturale Linguistik zusammengetragen hatte, ist hier Jahrzehnte lang vernachlässigt worden.

27) Aus diesem Grund ist der in der Orthographiedebatte und in der Schreibdidaktik immer noch geläufige Begriff der Laut-Buchstaben-Beziehung 
Tons. ${ }^{27)}$ Die Zeichengestalt des gesprochenen Wortes ist ,der' modulierte Ton. Der Singular ist hier wesentlich. ${ }^{28)}$

Die Schemata der Modulationsveränderung nennt man traditionellerweise Phoneme: In der orthodoxen Lesart der Phonologie gelten diese als Schemata der Artikulation von Lauten. Doch die Schwierigkeiten dieser Lesart werden schon beim Studium des klassischen Phonembegriffs deutlich. Der in der Phonologie geläufige Ausdruck „/p/“ denotiert realiter keineswegs das Schema eines „Lautes p“, sondern die Bewegung einer labialen Sprengung, die isoliert nie in der Sprache vorkommt, sondern immer nur als Initiale einer sich kontinuierlich fortsetzenden Artikulationsbewegung auftritt, welche mit vollzogener Sprengung einen höheren Sonoritätsgrad erzeugt. Schon der Prager Schule gilt die Silbe, nicht das Phonem als kleinste artikulatorische Einheit, und diese hat immer einen vokalischen oder mindest sonantischen Kern. ${ }^{29)}$

Im System der Alphabetschrift kann man diese kontinuierliche Modulation nur folgendermaßen darstellen: /pf/, /pr/, .., /pa/. ${ }^{30)}$ Doch das ist - immer unter der Perspektive des Exemplifizierens des Musters in Hinsicht auf seine Artikulationsweise betrachtet - in etwa so, als würde man die Bewegung des Umblätterns einer Buchseite durch die aufeinander folgende Wiedergabe beider Buchseiten darstellen:

$\mathrm{S}_{1} \mathrm{~S}_{2}$

Mehrfaches Umblättern wäre dann so darzustellen:

so irreführend. Die Kinder können gar nicht verstehen, was ihnen unter diesem Titel erklärt wird. Vielmehr müssen sie versuchen, sich aus dem Material, das ihnen präsentiert wird, den zutreffenden Reim zu machen, und diesen zugleich auf zwei verschiedenen Feldern. Man braucht sich nicht zu wundern, wenn dabei viele Kinder auf der Strecke bleiben.

28) Diese Einsicht ist schon bei W. von Humboldt klar ausgesprochen. Das Wort ist ihm der "Laut, der einen Begriff bezeichnet". Vgl. Grundzüge, GS V, S. 410, dazu Stetter 1997, S. 455 ff.

29) Vgl. Jakobson 1969, S. 96 f.

30) Die Schrägstriche / ... / sollen hier lediglich besagen, daß die durch sie eingeschlossenen Zeichen Darstellungen von Fragmenten oraler Artikulation sind. 
$\mathrm{S}_{1} \mathrm{~S}_{2} \mathrm{~S}_{3} \mathrm{~S}_{4} \mathrm{~S}_{5} \ldots$

So etwa "stellt" die Buchstabenfolge $g+e+b+e+n$ das gesprochene Wort /geben/ „dar“. In logischer Hinsicht kann man diese verquere Darstellungsweise hinnehmen, denn da geht es ausschließlich darum, einer bestimmten Wortbedeutung eine eindeutig ausdifferenzierte Zeichengestalt zu geben. Im Hinblick auf das Exemplifizieren wäre dagegen die Darstellung im Kern der Sache nicht korrekt, denn die Figurenfolge $\mathrm{S}_{1} \mathrm{~S}_{2}$ bezeichnet ja eine Artikulationsbewegung, nämlich eine Artikulationsveränderung. Also wäre die Figur $\mathrm{S}_{1} \mathrm{~S}_{2} \mathrm{~S}_{3} \mathrm{~S}_{4} \mathrm{~S}_{5}$ als Darstellung der oralen Artikulation nicht adäquat, sondern man müßte sie in erster Näherung ersetzen durch etwas wie

$$
\begin{aligned}
& \mathrm{S}_{1} \mathrm{~S}_{2} \\
& \mathrm{~S}_{2} \mathrm{~S}_{3} \\
& \mathrm{~S}_{3} \mathrm{~S}_{4} \\
& \quad \mathrm{~S}_{4} \mathrm{~S}_{5}
\end{aligned}
$$

Doch auch damit wäre das Problem ersichtlich nicht gelöst, sondern die Verlegenheit, es lösen zu können, nur noch deutlicher gemacht. Das bedarf hier keiner weiteren Begründung. ${ }^{31)}$ Die Graphik spricht für sich. Auch weitere graphische Bemühungen wie Klammerungen oder ähnliches würden das Problem nicht lösen können. Bewegung läßt sich im Medium der Schrift mimetisch nicht darstellen.

Darstellen könnten zwei nebeneinandergesetzte Figuren Bewegung nur dann, wenn man diese Figurenkonstellation ihrerseits bewegte. Vom Film her ist uns dieses Verfahren vertraut: die starren, neben-

31) Man braucht bloß, um dies zu sehen, an die Stelle der Variablen $S_{1}, S_{2} \ldots$ Buchstaben einsetzen:

$$
\begin{aligned}
& \mathrm{ge} \\
& \mathrm{e} \mathrm{b} \\
& \mathrm{b} \text { e } \\
& \quad \mathrm{en}
\end{aligned}
$$

Die Fragmentierung bleibt. Sie ist sozusagen nur von der horizontalen Ebene in die Zweidimensionale verschoben. 
bzw. hintereinander angeordneten Bilder können Bewegung darstellen, indem man sie bewegt. Also beruht die Fähigkeit von Zeichen, etwas mimetisch bzw. exhibitiv darzustellen, auf der internen Qualität ihres ,Seins“ als Gestalten, also auf dem, was man mit Peirce ihre Ikonizität nennen könnte. ${ }^{32)}$ Diese Eigenschaft bzw. Eigenschaftsdimension von Zeichen hat mit Ähnlichkeit und Unähnlichkeit der phänomenalen Erscheinung zu tun: Das Schwarz-weiß-Foto exemplifiziert, was es darstellt, in vielen Hinsichten, sicher auch durch Bezugnahme auf Helldunkel-Kontraste, ebenso sicher nicht durch Bezugnahme auf Farbkontraste oder Dreidimensionalität.

Dies wirft ein Licht darauf, daß das Arbitraritätsprinzip seinen Ursprung im Ästhetischen hat, auch wenn es bei Saussure selbst in einer in dieser Hinsicht irreführenden logischen Begrifflichkeit expliziert wird. ${ }^{33)}$ Es ist sozusagen der Mangel an Ähnlichkeiten und Differenzen der Gestalt, die geringe Prägnanz der Ikonizität, die das signe linguistique zum idealen Träger schematisierter Bedeutungen macht. Dies muß bei Zeichen, die technisch so oder so verfertigt werden können, in anderem $\mathrm{Maß}$ der Fall sein als bei Zeichen, deren Produktionsverfahren sie im Vollzug des Verfahrens konstituiert. Daher ist dem gesprochenen Wort der Charakter der Geste nie zu nehmen. Daher ist andererseits das Alphabet, dessen Zeichenrepertoire sich vollständig auf drei Figuren zurückführen läßt - auf Kreis, Halbkreis und Gerade -, dessen Ikonizität also auf ein Minimum an Prägnanz reduziert ist, das ideale Medium der Darstellung „reiner“ Bedeutungen und einer durch Bildlichkeit nicht behinderten Metaphorik - etwas, wozu Han ze oder Kanji bei aller Jahrtausende währenden Konventionalisierung nie gelangen. ${ }^{34)}$ Schon die ungleich größere Anzahl und die Vielfältigkeit möglicher Arrangements der "Striche“ sichern ihnen ein höheres Maß an Ikonizität.

32) Vgl. Ch. S. Peirce, Collected Papers 5.286 ff., dazu Stetter 1983, S. 284 ff.

33) Vgl. EC fasc. 2, al. 1121 ff., IIIC 280 ff. Zum Problem der Arbitrarität vgl. auch Stetter 1997, Kap. 4.

34) Dies kann natürlich ebenso ein Vorzug sein. Z. B. dürfte die weitaus bessere Lesbarkeit der Han ze und Kanji im Vergleich zu in Alphabetschrift geschriebenen Wörtern etwas mit ihrer hohen ikonischen Prägnanz zu tun haben. 
Dies ist das kategoriale Problem, das mit der sogenannten Transkription $^{35)}$ verbunden ist. Schon der Name der Operation ist irreführend. Transkribieren kann man, genau genommen, nur von einem Schriftsystem ins andere. Man kann Kanji in Kana und diese in Romaji transkribieren. Es geht, genauer gesagt, um die exhibitive Darstellung der Zeichen eines Sprachmediums in einem anderen Medium, um das, was wir den ,mimetischen' Gebrauch der Schrift genannt hatten.

Will man Klarheit über dieses Problem gewinnen, so muß man versuchen, die Beschreibung der medienspezifischen ästhetischen Eigenschaften der sprachlichen Zeichen, die wir oben begonnen hatten, zu präzisieren. Die Linguistik - sofern sie sich zu der Position durchringt, da $\beta$ jedweder Begriff sprachlicher Kompetenz nur medienspezifisch $z u$ formulieren ist - gewinnt eine neue Aufgabe bzw. Dimension: die Ästhetik der von ihr beschriebenen Zeichen. Erst damit wird man die Alphabetzentriertheit der klassischen Linguistik überwinden können. Der einfachste Fall ist wohl eine Ästhetik der schriftlichen Sprachelemente. Daß Buchstaben wie andere Schriftelemente, Kanji, Kana usw., als dauerhaft gegenüber der Flüchtigkeit der Oralität gekennzeichnet

35) Vgl. hierzu Stetter 1997, S. 126 ff. Jäger (2000b) spricht von Transkription in übertragenem Sinn: einmal im Sinne reflexiver, metasprachlicher Kommentierung von Äußerungen - er nennt dies ein „intramediales“ Verfahren -, zweiten im Sinne der „Kommentierung, Erläuterung, Explikation und Übersetzung (der Semantik)“ eines medialen Systems durch ein zweites, also ein „intermediales“ Verfahren (S. 8). Beide Verfahren dienen nach Jäger dem "Lesbarmachen des jeweils thematisierten symbolischen Sytems“ (ebd.). Es ist klar, daß es hier erstens nicht um die Exemplifizierung von Zeichengestalten geht - das macht insbesondere der Hinweis auf die Semantik deutlich - und daß zweitens die Rede von Symbolsystemen metaphorisch zu verstehen ist, im Sinne von Spachspiel-Ausschnitten, langage-Phänomenen o. ä., denn wenn das Resultat einer Transkription in Jägers Sinn ein Skript, d. h. „lesbar gemachter Ausschnitt" des betreffenden "thematisierten symbolischen Systems" ist, mithin ein effektiv erzeugter, logisch linearisierter Text, dann muß es sich bei dem, was da transkribiert wurde, um eine Entität derselben kategorialen Ordnung handeln. Unter einem System versteht man - jedenfalls in der Regel - jedoch nicht solche effektiv erzeugten Performanzausschnitte, sondern solchen zugrunde liegende Regeln oder Analogien, die dem betreffenden Performanzausschnitt seine Form geben. 
werden, greift - das haben die Überlegungen zum mimetischen Schriftgebrauch wohl definitiv gezeigt - zu kurz. Auch Bewegungen können ja durchaus dauerhaft sein. Schriftliche Zeichen sind ,starr, ein für allemal in einem formatierten Raum gegeben. ${ }^{36)}$ Das ist ihre grundlegende phänomenale Eigenschaft, aus der alle weiteren folgen. Auch wenn die Tinte, mit der ein Buchstabe geschrieben wurde, mit der Zeit vergilbt - solange er noch gelesen werden kann, handelt es sich, auch nach Hunderten von Jahren, noch immer um denselben Buchstaben. Dasselbe gilt, wenn das Papier oder Pergament an der betreffenden Stelle beschädigt und dadurch seine Gestalt teilweise zerstört worden sein sollte. Solange anhand der verbleibenden Fragmente eindeutig erkennbar ist, um welchen Buchstaben es sich handelt, existiert er an dieser Stelle, und zwar ganz, nicht fragmentarisch. Also gehört Redundanz mit zu den grundlegenden phänomenalen, d. h. ästhetischen Eigenschaften schriftlicher Zeichengestalten - und zweifellos auch zu deren oralen oder gebärdensprachlichen Pendants, ${ }^{37)}$ und sie organisiert sich medienspezifisch verschieden.

Auch wenn es kursive Formen schriftlicher Zeichen gibt, so sind diese doch grundsätzlich, mit N. Goodman zu sprechen, endlich differenzierbar und disjunkt. ${ }^{38)}$ Den Beleg dafür liefern schon ältere Typenradschreibmaschinen, mit denen es möglich war, kursiv verbundene Wörter mechanisch zu schreiben. ${ }^{39)}$ Gerade der Fall „eigentlich“, näm-

36) Am schönsten ist diese Eigenschaft wohl in der elaborierten japanischen Kalligraphie exemplifiziert, wo das Resultat der Schreibbewegung oft mehr deren Geste als die Figur des betreffenden, durch sie geschriebenen Zeichens festhalt. Gerade dies macht es ja möglich, Nichtsagbares zu schreiben. Vgl. hierzu Shimano und Tani 1990.

37) Analoge Beobachtungen lassen sich ja in der mündlichen oder in der Gebärdensprache ohne Schwierigkeiten ausweisen. Redundanz ist traditionell als informationstheoretisches Problem behandelt worden. Es wäre aber zum besseren Verständnis der Funktionsweise natürlicher Sprachen sinnvoll, sie kategorial dort unterzubringen, wo sie ihre Funktion entfaltet, und das ist die Ästhetik der Gestalten.

38) Vgl. Goodman 1997, Kap. 4: Die Theorie der Notation, S. 125 ff.

39) Bei den „kursiv“ genannten Varianten der Fontsätze von Textverarbeitungsprogrammen handelt es sich natürlich nicht um Kursivschrift im eigentlichen Wortsinn. 
lich handschriftlich kursiv geschriebener Formen, der dieser These ja auf den ersten Augenschein hin widerspricht, jedenfalls was die endliche Differenzierbarkeit angeht, ist hier von Interesse, z.B. der der mittelalterlichen Ligaturen, die sich noch im Typenrepertoire Gutenbergs finden. ${ }^{40)}$ Es ist die Starrheit dieser Formen - nämlich qua geschriebener ${ }^{41)}$-, die es möglich macht, sie im Laufe der Zeit so zu bearbeiten, daß die signifikanten Züge dieser Gestalten, die sie von anderen unterscheiden, mehr und mehr schematisiert werden, bis schließlich der Typ gefunden ist, den man in einem formalen System von Maßangaben definieren kann. ${ }^{42)}$ So wird er auf Dauer starrgestellt. Die Buchstaben der lateinischen Antiqua haben sich als Typen bis heute nicht, jedenfalls nicht wesentlich verändert - etwas, das im Bereich der „beweglichen“ Sprachmedien undenkbar wäre.

$\mathrm{DaB}$ mit verschiedenen Techniken ein und dieselbe Gestalt erzeugt werden kann, erweist sich auch aus dieser Perspektive als für die Ästhetik schriftlicher Sprachzeichen relevant. Diese Zeichen sind in der Tat regelbar und ihre Erzeugung damit in einem Maß konventionalisierbar, der für die „naturwüchsigen“ Medien der oralen oder der Gebärdensprache undenkbar wäre. ${ }^{43)}$ Dies gilt für Kanji ebenso wie für Buchstaben. Eine überraschende Konsequenz, die aber unabweisbar ist: das Arbitraritätsprinzip muß offenbar für die Schriftsprache ganz anders

40) Vgl. hierzu Giesecke 1990.

41) D. h. sie sind Elemente der Textur, nicht des Textes. Text ist, was geschrieben und verstanden wird, Textur, was geschrieben ist und gelesen wird. Vgl. hierzu Stetter 1997, Kap. 7.

42) Am einfachsten in einem hinreichend feinen System von Rasterpunkten. Man denke etwa an das mittlerweile erreichte Auflösungsvermögen von digitalen Kameras. Es ist für eine Digitalisierung des Kursiven nicht notwendig, in der handschriftlichen Darstellung des Wortes, z. B. in, den Punkt angeben zu können, wo das $i$ aufhört und das $n$ beginnt. Es genügt zu wissen, daß dieses Wort in zwei Buchstaben geschrieben ist bzw. wird.

43) Auch hier ein kategorialer Shift zwischen Mündlichkeit und Schriftlichkeit, der für die Didaktik der Literalisierung Konsequenzen haben müßte. Kinder können keinen Begriff eines geregelten Sprachgebrauchs haben - was nota bene etwas völlig anderes ist als ein ,regelmäBiger' Sprachgebrauch. (Autofahrer verhalten sich in einem sich bildenden Stau normalerweise ganz regelmäßig - dadurch entsteht ja oft erst der Stau -, man wird aber doch nicht sagen können, ihr Verhalten sei geregelt.) 
formuliert werden als für die orale und - vermutlich - für die Gebärdensprache. ${ }^{44)}$

Wir können also die wichtigsten ästhetischen Merkmale schriftlicher Sprachzeichen mit Begriffen wie Konstanz, nichtprozedural (starr), lokal identifizierbar, endlich differenzierbar, disjunkt usw. wenigstens vorläufig andeuten. ${ }^{45)}$

Ganz anders dagegen die Elemente der oralen und der Gebärdensprache: Ihre ästhetischen Eigenschaften muß man mit Begriffen wie Fluktuan $z^{46)}$, prozedural (beweglich), temporal identifizierbar, kontinuierlich usw. umschreiben. Der vielleicht wichtigste Unterschied gegenüber den schriftlichen Zeichen liegt wohl darin, daß die Technik der Artikulationsweise nicht willkürlich veränderbar ist, und dies aus zwei Gründen: erstens wird sie nicht erlernt, sondern im primären Spracherwerb erworben - in einer Weise, die bis heute im dunkeln liegt ${ }^{47)}$-,

44) Eine Hinsicht, in der dies zu geschehen hat - die in anthropologischer Hinsicht vermutlich wichtigste -, kann ich hier nur andeuten: die Rückkopplung der schriftlichen Zeichen ans Gefühl. In Hinblick auf die Einbildungskraft hatte dies W. von Humboldt bereits in aller Klarheit gesehen: „... so kann die Übertragung des erst als Ton gehefteten Gedankens auf einen Gegenstand des Auges, nach Massgabe der Art, wie sie geschieht, dem Geist sehr verschiedene Richtungen geben." (Buchstabenschrift, WW III, S. 84 f.). Entsprechendes gilt aber auch für die Verankerung der Sprachartikulation im Gefühl. Wenn Zeichengestalten mechanisch in einer Weise erzeugt werden, die materialiter nur noch vom visuellen Sinn kontrolliert wird, kann das Resultat dieses Prozesses nicht in gleicher Weise ins Körpergefühl integriert werden, das ja immer an Bewegung gekoppelt ist, wie die mündliche oder die gebärdensprachliche Artikulation. Jeder Fremdsprachendidaktiker weiß aber, wie wichtig es für das Erlernen einer Fremdsprache ist, ein Gefühl für die Artikulationsweise dieser Sprache zu gewinnen.

45) $\mathrm{Daß}$ es sich bei diesen ästhetischen Merkmalen um Merkmale einer anderen kategorialen Ordnung handelt als bei den sogenannten distinktiven Merkmalen der Phonologie, dürfte klar sein: Sie sind distinktiven Merkmalen logisch vorgeordnet, bereiten den Boden vor, auf dem solche dann definiert werden können.

46) Vgl. hierzu Stetter 1997, S. 129 ff.

47) Daß die Artikulationsfähigkeit als Implikat der Sprachfähigkeit angeboren ist, darüber besteht kein Zweifel. Welcher Art aber die kognitiven Prozesse sind, die ablaufen, wenn das Kind "sprechen lernt", dies ist bis 
zweitens ist diese Technik der Artikulationsweise dem Prozeß der Zeichenbildung nicht äußerlich, sondern interner Bestandteil desselben. Denn das gesprochene Wort ist - anders als das geschriebene - stets auch Geste. ${ }^{48)}$ Das gebärdensprachliche sogar buchstäblicherweise.

Diese wenigen Bemerkungen müssen hier genügen, die Problemstellungen einer linguistischen Ästhetik anzudeuten. Es bleibt mit Blick auf des Problem des mimetischen Sprachgebrauchs, von dem wir ausgegangen waren, zu fragen, was passiert, wenn ein prozeduraler Sprachmodus durch einen ,starren' exemplifiziert wird. Hinsichtlich der Logik ist dieser "Medienbruch“ offensichtlich nicht oder nur unerheblich von Bedeutung. Schon die fragmentarischen Überlegungen, die wir zur Ästhetik der linguistischen Zeichenartikulation angestellt haben, verdeutlichen indessen, daßmit diesem Medienbruch Eigenschaften der oralen Sprache, die in rhetorischer oder ästhetischer Hinsicht relevant sind ihr Klang ${ }^{49)}$, die Intonation, natürlich die Sprechgeschwindigkeit etc. systematisch ausgeblendet werden. ${ }^{50)}$ Es zeigt sich hier deutlich, in welchem Maß sich die unverkennbar logizistische Perspektive der Linguistik - die Fixierung auf den Satzbegriff, ${ }^{51)}$ die Funktion der soge-

heute jedenfalls noch weitgehend unerforscht. Die neuere neurologische Forschung widerspricht hier der Annahme Chomskys, daß sich Spracherwerb als gleichsam selbsttätige Entwicklung angeborener Sprachmodule vollzieht. Vgl. hierzu Spitzer 1996, S. 229 ff.

48) $\mathrm{Ob}$ das geschriebene Zeichen groß oder klein, dick oder dünn, deutlich oder undeutlich geschrieben oder gedruckt ist, ist gleichgültig, solange man es nur lesen kann. Für das orale Wort ist es dagegen keineswegs gleichgültig, ob es laut oder leise, schnell oder langsam usw. artikuliert wird. Das haben schon die alten Rhetoren gewußt und daher die actio als eigenen - und wichtigen - Teil der Disziplin entwickelt. Vgl. hierzu Quintilian [1988], 2. Teil, 11. Buch, S. $609 \mathrm{ff}$.

49) Jede Sprache hat ja ihren spezifischen Klang, der sich aus der Ökonomie und Nutzung ihres Phoneminventars ergibt. Das Castillano z. B. klingt deutlich anders als das Katalanische, so eng verwandt beide Sprachen auch sind.

50) Mit der Gebärdensprache dürfte es sich analog verhalten. Diese Dimensionen sind aber bislang kaum erforscht.

51) Dieser verdankt sich ja ganz und gar der Grundlegung der formalen Logik in Aristoteles' Peri hermeineias und in der Kategorienschrift. Vgl. hierzu Stetter 1997, Kap. 9. 
nannten ,Logischen Form' in der generativen Syntax usw. - der schriftlichen, und zwar der alphabetschriftlichen Praxis der Linguistik verdankt.

Damit stehen wir allerdings vor einem Dilemma: Die Linguistik braucht die verschriftlichende Exemplifizierung von Elementen nichtschriftlicher (d. h. fluktuierender, prozeduraler, ...) Sprachmodi, denn ihr Erkenntnisinteresse geht auf die Beschreibung, Explikation, Erklärung, ... von Typen. Zur Beschreibungsadäquatheit gehört aber offenbar - wie wir gesehen haben - wesentlich auch die zutreffende mimetische Darstellung dieser Modi als prozeduraler. Denn wenn die langue nicht nur als trésor mental, sondern als produktives Kompetenzsystem begriffen und theoretisch rekonstruiert werden soll, dann müssen dabei auch diejenigen ihrer Eigenschaften erfaßt werden, die für die Bildung der parole in rhetorischer oder ästhetischer Hinsicht bedeutsam sind. Doch ohne verschriftlichende Verfahren ist systematische Sprachbeschreibung unmöglich. Die Lösung dieses Dilemmas muß wohl auf verschiedenen Ebenen gesucht werden:

(1) der einer adäquaten phänomenalen Repräsentation des Einheitenbestandes der verschiedenen medialen Modi einer Sprache. Man wird prozedurale schriftliche Darstellungsformen nichtschriftlicher Sprachmodi entwickeln müssen, z. B. multimediale Computersimulationen von oraler oder von Gebärdensprache. ${ }^{52)}$

(2) auf der Ebene einer Erweiterung des klassischen Kompetenzbegriffs, die rhetorisch und ästhetisch relevante Aspekte der Sprachproduktion ebenso erfaßt wie die logischen, und dies in allen medienspezifischen Sprachmodi. Man wird sich vom Satz als Grundkategorie der Syntax verabschieden müssen. Diesen Status verdankt er unzweifelhaft seiner logischen Herkunft. Die Phrase, nicht der Satz ist die Domäne der Linguistik. ${ }^{53)}$

(3) Auf der Ebene der für die Linguistik konstitutiven theoretischen

52) Wegweisend dabei könnte Jakobsons ingeniöser Versuch sein, die Klangcharakteristika phonematischer Artikulationen auf Farbskalen abzubilden. Vgl. Jakobson 1969, S. 114 ff. Dieser Versuch bewegte sich noch im "Starren“. Man muß die Grundidee, die dahinter stand, auf das heutige technische Niveau übersetzen.

53) Diesen Gedanken habe ich systematisch entwickelt in Stetter 2001. 
Grundunterscheidungen. Hier muß das Verhältnis von Kompetenz und Performanz neu überdacht werden. Die Konzentration auf das Studium der Kompetenz, besser gesagt: die Vernachlässigung der Performanz muß überwunden werden. Denn geht man davon aus, daß sich ein linguistischer Kompetenzbegriff medienneutral nicht zutreffend formulieren läßt, ${ }^{54)}$ dann muß es sich bei dem Verhältnis beider Instanzen um ein Rückkopplungsverhältnis handeln, so wie Saussure schon das Verhältnis von langue und parole gesehen hatte. ${ }^{55)}$ I. ü. wird dies auch von der neurologischen Erforschung der Sprache nahegelegt. ${ }^{56)}$

Damit wird auch aus dieser Perspektive die Architektur des Modells in Frage gestellt, das spätestens seit Chomskys Aspects die theoretische Debatte der Linguistik bestimmt hat. Wenn die ästhetische Formung sprachlicher Ausdrücke integraler Bestandteil des Sprachprozesses ist - um diesen Begriff hier als Verallgemeinerung der saussureschen Kategorie der parole zu nehmen - und damit Bestandteil einer jeden adäquaten linguistischen Beschreibung, dann läßt sich die Annahme eines ,autonomen' Syntaxmoduls, dessen Output durch eine phonologische Komponente in eine ,willkürliche einzelsprachliche Form übersetzt wird, nicht halten. ${ }^{57)}$ Es geht nicht nur um die Revision der Unterscheidung von Tiefen- und Oberflächenstruktur. Die war ja schon im Minimalistischen Programm fraglich geworden. Die die gesamte generative Linguistik charakterisierende hiatische Trennung von Form und Inhalt des sprachlichen Zeichens wird hinfällig. Damit gewinnt die semiologische Position F. de Saussures für die „postchomskysche" Linguistik neue Aktualität. ${ }^{58)}$

Namentlich gibt die "Wiederentdeckung“ der Sprachästhetik als eines konstitutiven Bestandteils systematischer Sprachbeschreibung ${ }^{59)}$ der insbesondere von S. Krämer und L. Jäger vorgetragene These, daß der linguistische Kompetenz-Begriff medienneutral nicht $\mathrm{zu}$ formulieren

54) Vgl. hierzu Jäger 2000a und Stetter 2001.

55) Vgl. CLG S. $27 \mathrm{ff}$.

56) Vgl. hierzu Spitzer 1996, S. $229 \mathrm{ff}$.

57) Dies gilt für das sogenannte Prinzipien-und-Parameter-Modell i. ü. ebenso wie für das "Minimalistische Programm“. Auch dieses kennt ja noch eine ,Phonologische Form:

58) Vgl. hierzu Stetter 2001. 
sei, ${ }^{60)}$ ihre materiale Grundlage, übersetzt sie auf die Ebene der linguistischen Theoriebildung. Die hieraus sich zwingend ergebende semiotisch-semiologische Reformulierung linguistischer Grundbegriffe habe ich in einigen Grundzügen in einer anderen Arbeit entwickelt, namentlich bezüglich der Konzepte, mit denen der Begriff der Konstituenz linguistisch konstruiert wird. ${ }^{61)}$ Wie eine linguistische Ästhetik aufzubauen ist, ist vorerst im einzelnen noch schwer abzusehen. Ihre Funktion allerdings ist klar: Sie wird die verschiedenen Beschreibungsebenen der Phonologie/Graphematik, ..., Morphologie und Syntax zu integrieren haben und von ihr aus wird man das Verhältnis von langue und parole bzw. von Kompetenz und Performanz neu zu überdenken haben. Denn die ästhetische Erscheinung welches sprachlichen Ausdrucks auch immer ist per se eine Einheit, die alle artikulatorischen Ebenen umfaßt, und sie ist eine Ganzheit, die sich im Vollzug der Artikulation bildet und die auch nur performativ, zuhörend oder lesend, wahrgenommen werden kann. ${ }^{62)}$ Sie gehört der Ordnung des Textes an, nicht der der Textur. ${ }^{63)}$

59) Von "Wiederentdeckung“ ist hier in der Tat zu reden: Wie so vieles andere ist auch der Gedanke einer sprachwissenschaftlichen Ästhetik schon bei W. von Humboldt nicht nur angedeutet, sondern ausgeführt was i. ü. bei der Herkunft der Sprachphilosophie und -theorie Humboldts aus der kantischen Ästhetik nicht überrascht. Vgl. hierzu Stetter 1997, Kap. 10.

60) Vgl. hierzu Krämer 1998 und Jäger 2000a.

61) Vgl. Stetter 2001.

62) Für die Gebärdensprache gilt dies analog.

63) Ansatzweise machen dies Publikationen der visuellen Poesie deutlich. Vgl. hierzu Gomringer 1996 und Schmidt 1996. 


\section{Literatur:}

Austin, John L. (1975): How to Do Things with Words. 2nd ed. Oxford: Peregrine Books.

Austin, John L. (1979): Zur Theorie der Sprechakte. Deutsche Bearbeitung von Eike von Savigny. 2. Aufl. Stuttgart: Reclam.

Eisenberg, Peter (1989): Grundriß der deutschen Grammatik. 2. Aufl. Stuttgart: Metzler.

Giesecke, Michael (1990): Orthotypographia. Der Anteil des Buchdrucks an der Normierung der Standardsprache. In: Christian Stetter (Hg.) 1990, S. $65-89$.

Gomringer, Eugen, Hg. (1996): visuelle poesie. anthologie von eugen gomringer. Stuttgart: Reclam. (= reclam 9351)

Goodman, Nelson (1984): Weisen der Welterzeugung. Frankfurt a. M.: Suhrkamp (= stw 863).

Ders. (1997): Sprachen der Kunst. Entwurf einer Symboltheorie. Frankfurt a. M.: Suhrkamp (= stw 1304)

Grewendorf, Günther, Hamm, Fritz und Sternefeld, Wolfgang (1987): Sprachliches Wissen. Eine Einführung in moderne Theorien der grammatischen Beschreibung. Frankfurt a. M.: Suhrkamp.

Humboldt, Wilhelm von [1968]: Gesammelte Schriften. 17 Bde. Hg. von Albert Leitzmann u.a. Berlin 1903ff Photomech. Nachdr. Berlin 1968. Zitiert als GS.

Jäger, Ludwig, Hg. (1979): Erkenntnistheoretische Grundfragen der Linguistik. Stuttgart: Kohlhammer.

Ders. (2000a): Medialität und Mentalität. Die Sprache als Medium des Geistes.

(Typoskript).

Ders. (2000b): Transkriptivität. Zur medialen Logik der kulturellen Semantik. (Typoskript).

Jakobson, Roman (1969): Kindersprache, Aphasie und allgemeine Lautgesetze. Frankfurt a. M.: Suhrkamp.

Krämer, Sybille (1998): Das Medium als Spur und als Apparat. In: Dies., Hg. (1998): Medien, Computer, Realität. Wirklichkeitsvorstellungen und Neue Medien. Frankfurt a. M.: Suhrkamp. S. $73-94$.

Dies. (2000): Über den Zusammenhang zwischen Medien, Sprache und Kulturtechniken. In: Kallmeyer, Werner, Hg. (2000): Sprache und neue Medien. Berlin, New York: de Gruyter. S. $31-56$.

Peirce, Charles Sanders [1931 ff.]: Collected Papers. Vols. I - VI ed. by Charles Hartshorne and Paul Weiss. Cambridge/Mass. 1931 - 1935. Vols. VII VIII ed. by W. Burks. Ibid. 1958: Belknap Press. Zitiert als Collected Papers.

Quintilianus, Marcus Fabius [1988]: Ausbildung des Redners (institutio orato- 
ria). Hgg. und übers. von Helmut Rahn. 2., durchges. Aufl. Darmstadt: Wiss. Buchges.

de Saussure, Ferdinand (1972): Cours de linguistique générale. Édition critique préparee par Tullio de Mauro. Paris: Payot. Zitiert als $C L G$.

Schmidt, Siegfried J., Hg. (1996): „ersichtlichkeiten“. internationale visuelle texte der 90er. Siegen: Uni-GHS Siegen.

Schneider, Hans J. (1992): Phantasie und Kalkül. Über die Polarität von Handlung und Struktur in der Sprache. Frankfurt a. M.: Suhrkamp.

Shimano, Eido Tai, und Tani, Kogetsu, (1990): Zen Wort, Zen Schrift. Zürich und München: Theseus.

Spitzer, Manfred (1996): Geist im Netz: Modelle für Lernen, Denken und Handeln. Darmstadt: Wissenschaftliche Buchgesellschaft.

Stetter, Christian (1983): Peirces semiotische Schemata. In: Eschbach, Achim und Trabant, Jürgen, Hgg. (1983): History of Semiotics. Amsterdam/ Philadelphia: John Benjamins Publ. S. 277 - 310.

Ders., Hg. (1990): Zu einer Theorie der Orthographie. Interdisziplinäre Aspekte gegenwärtiger Schrift- und Orthographieforschung. Tübingen: Niemeyer.

Ders. (1997): Schrift und Sprache. Frankfurt a. M.: Suhrkamp.

Ders. (1999a): Der Käfer in der Schachtel: Das Privatsprachenproblem und die Universalgrammatik. In: Lili 29, 1999, Heft 115, S. $37-66$.

Ders. (2001): Am Ende des Chomsky-Paradigmas - zurück zu Saussure? (Erscheint in: Cahiers Ferdinand de Saussure 54, 2001). 